\title{
Current of Child Sexual Abuse in Asia: A Systematic Review of Prevalence, Impact, Age of First Exposure, Perpetrators, and Place of Offence
}

\author{
Tetti Solehati ${ }^{1{ }^{2 *}}$, Iqbal Pramukti ${ }^{3}$, Yanti Hermayanti ${ }^{2}$, Cecep Eli Kosasih ${ }^{4}$, Henny Suzana Mediani ${ }^{5}$ \\ ${ }^{1}$ Doctoral Study Program, Faculty of Medicine, Universitas Padjadjaran, Sumedang, West Java, Indonesia; ${ }^{2}$ Department of \\ Maternity, Faculty of Nursing, Universitas Padjadjaran, Sumedang, West Java, Indonesia; ${ }^{3}$ Department of Community Health \\ Nursing, Faculty of Nursing, Universitas Padjadjaran, Sumedang, West Java, Indonesia; ${ }^{4}$ Department of Critical Nursing, \\ Faculty of Nursing, Universitas Padjadjaran, Sumedang, West Java, Indonesia; ${ }^{5}$ Department of Pediatric Nursing, Faculty of \\ Nursing, Universitas Padjadjaran, Sumedang, West Java, Indonesia
}

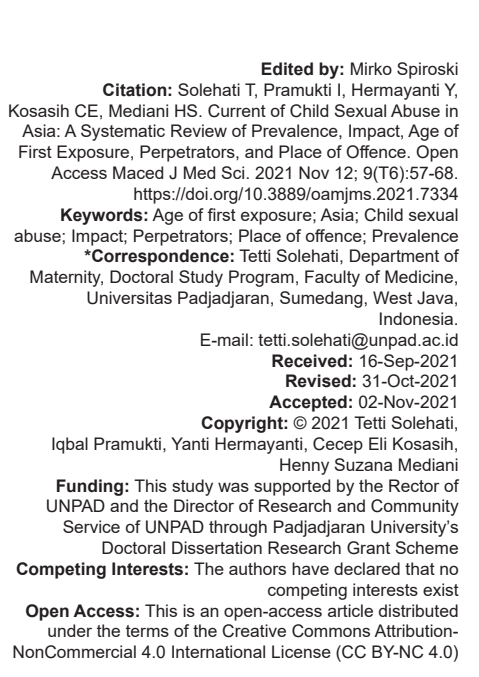

\section{Abstract}

BACKGROUND: Child sexual abuse (CSA) is a serious global problem and challenge for all people because it increases the risk of various issues.

AIM: This study investigated the CSA in Asian countries focusing on prevalence rates, impact, victim's age of first exposure, type of CSA, perpetrator, and places of CSA offence.

METHODS: We searched PubMed, Medline, ProQuest, ScienceDirect, CINAHL, Academic Search Complete, ClinicalKey, PsycINFO, Google Scholar, and manual search for studies published between January 2011 and November 2020. Only articles related to CSA in Asia were included in this review.

RESULTS: The results showed that prevalence of CSA ranged from $2.2 \%-94 \%$ for girls and $1.7 \%-49.5 \%$ for boys. The prevalence rates for non-contact abuse were $12.6 \%-56.5 \%$ for girls and $0.7 \%-68.7 \%$ for boys; contact abuse was $5.3 \%-67.2 \%$ for girls and $2.2 \%-53.3 \%$ for boys; penetrating abuse was $0.5 \%-88.24 \%$ for girls and $1.7 \%-57.1 \%$ for boys. The findings reported most victims' age of first exposure was preteen. The rate of the violation occurring in the victim's house was $6.1 \%-41.9 \%$; most CSA perpetrators are known by victims. This study reported adverse impacts on CSA victims such as psychological, health, and physical disorders, and changes in behavior. In addition, other findings were found, including recovery, perceptions, values, causes, and expectations of victims of CSA.

CONCLUSIONS: The incidence of CSA in Asia is still high and the age of the first victims is early adolescence. The majority of victims are women who already know the perpetrator, and taboo cultural factors are thought to play a role in increasing CSA in Asia. The results indicate the need to develop CSA prevention efforts that involve culture.

\section{Introduction}

Children are a very important national asset and they have the right to be protected from all forms of harassment [1], including child sexual abuse (CSA). CSA is defined as the participation of children in a sexual activity that violates the law or social taboos of society in which the child does not understand and cannot give consent to the activity. The activity is carried out by adults and children using the forced coercion of children [2], [3], [4]. There are three forms of CSA, namely: non-contact abuse (exposure to sexual activity that does not involve physical contact), contact abuse (sexual touch), and penetrating abuse [5], [6]. Children are a group at risk of experiencing sexual abuse from the age of 0-18 years. An increased risk of sexual abuse occurs just before puberty when the child enters early adolescence [6]. CSA researches in 14 countries found that at least $10 \%$ of boys and $15 \%$ of girls had experienced sexual abuse in their childhood [7], [8]. CSA was a serious challenge for all people in the world because it increased the risk of various problems, both mental and physical [9], [10]. CSA is a serious chronic and global problem widespread in society [5], [11], [12], [13], [14], [15], [16], [17], [18]. The impact on victims is not only during childhood but after becoming adults, and throughout maybe even their whole life, leading to problems such as depression [19], post-traumatic stress disorder [20], risky sexual behaviors [21], and going on to become the perpetrator of the abuse cycle [22]. CSA is a global problem that has a long-lasting negative impact on children's lives.

A review of Stontenborgh's systematic research on CSA around the world showed that CSA levels were alarming. The research in Asia found high rates of CSA for girls (11.3\%) and boys (4.1\%) [12]. The results of a meta-analysis study in China found that 
the prevalence of CSA was about $15.3 \%$ in girls while $13.8 \%$ in boys [23]. The prevalence of CSA tended to be higher in girls than boys [6], [17], [24], and other studies reported the opposite [25]. There were also studies which reported that the prevalence of CSA was equally high in girls and boys [16]. The true prevalence of CSA was likely to be higher than reported [26], as families may not report due to stigma and the implications on the safety of their children, mistrust of the police, and the presence of perception damaging the reputation of the whole family [27], [28]. Official statistical data may report that the numbers of CSA cases were under-represented because they were not reported or detected, have less safe and confidential assessments, and are not consistent with criteria for measurement of CSA [18], [29]. A study in India revealed that $53.2 \%$ of children experienced some form of CSA but the majority was not reported [25]. Reporting is not done because there are many contributing factors, one of which is the negative stigma that children and families will carry when other people find out that their child is a victim. Research on CSA in developing countries is still lacking [30], including CSA in Asia where data is still limited [31], and CSA incidents are reported to be increasing worldwide [26]. Previous literature studies of CSA have focused more on developed countries investigating the prevalence rate [17], [32], [33]. Therefore research on the prevalence of CSA was a challenge [18], especially in Asia. The need to estimate the prevalence of CSA in Asia is very important for health research in Asia, especially to allocate economic resources in health care.

From the description above, it can be concluded that CSA data in Asia is still limited; meanwhile, the number of CSA incidents is reported to increase every year so research on CSA becomes a challenge. A systematic review of the estimated prevalence rate, victims' age of first exposure, type of sexual abuse, perpetrator and relationship to the victim, place of offence, and impact of CSA in Asia is important and necessary for child health research in Asia and for the provision of support. It is also necessary to obtain information for researchers to understand the nature and magnitude of the problem to develop an appropriate strategy in prevention for guideline developers and policy makers, as well as observing progress and evaluating the effectiveness of CSA prevention strategies (Hobbs, 2005; Tanaka et al.,) CSA [34], [35]. With this systematic review research, it is hoped that the public will be open about CSA currently happening in Asia. The objectives of this study were to investigate and increase the understanding of CSA in Asian countries focused on prevalence rates, victims' age of first exposure, type of CSA, place of offence, perpetrator and relationship to the victim, impact of CSA, and identifying gaps in the current research.

\section{Methods}

\section{Search strategy for relevant study}

Using the guidelines of Preferred Reporting Items for Systematic Reviews and Meta-Analyzes (PRISMA) [36] in this systematic review, databases searched for research between 2011 - 2020 included PubMed, Medline, ProQuest, ScienceDirect, CINAHL, Academic Search Complete, ClinicalKey, PsycINFO, and Google Scholar. Searches were carried out using medical subject headings (MeSH) and the following keywords: "sexual abuse" OR "sexual violence" AND" Child sexual abuse" OR "Child sexual violence "AND "Prevalence" AND "Asia", using nine databases with same search strategy. In addition, for research questions, inclusion criteria, searching strategies, searching terms, searching engines, and study protocols were consulted with experts in the fields of children, sexual, reproductive health, and emergency of nursing (who are members of the author).

A study was eligible if it followed the following inclusion criteria in this study: 1) publication in peer reviewed scientific journals; 2) about sexual abuse experienced before the age of 18 years; 3) contained an estimated prevalence of CSA for girls and boys in Asian countries; 4) estimated the age of first exposure of CSA; $5)$ estimated type of CSA; 6) estimated perpetrator of CSA; 7) estimated relationship to the victims; 8) estimated places of offence; 9) estimated impact of CSA; 10) contained adequate primary data derived from cross-sectional, longitudinal, cohort, and case control studies with qualitative and mixed methods; 11) included articles in which CSA data was collected retrospectively from adults; 12) issued between January 2011 November 2020; 13) English language; 14) full text; 14) not a dissertation, letter epidemiological review, metaanalyze, systematic review, comment, review literature, book, book chapters, proceedings, or editorial.

\section{Study Selection}

We identified and filtered abstracts of all articles in the database following the inclusion criteria. An initial search of CSA articles in Asia from an electronic literature database yielded 13,539 titles. After excluding 829 duplicates, 12,710 articles remained. Then, after removing the review of title and abstract review, 41 articles remained for full text screening. Of the 41 articles, 5 articles were excluded on the grounds that they had low quality, and so only 36 articles met the requirements (Figure 1).

Each article was identified as relevant to the CSA in Asia by two of the authors who submitted the full text review and data extraction. The first and fourth authors reviewed abstracts of all articles identified in the database. All data was obtained in electronic form. Then, the second, third, fourth, and fifth authors reviewed the full text of the filtered article to assess and confirm its 


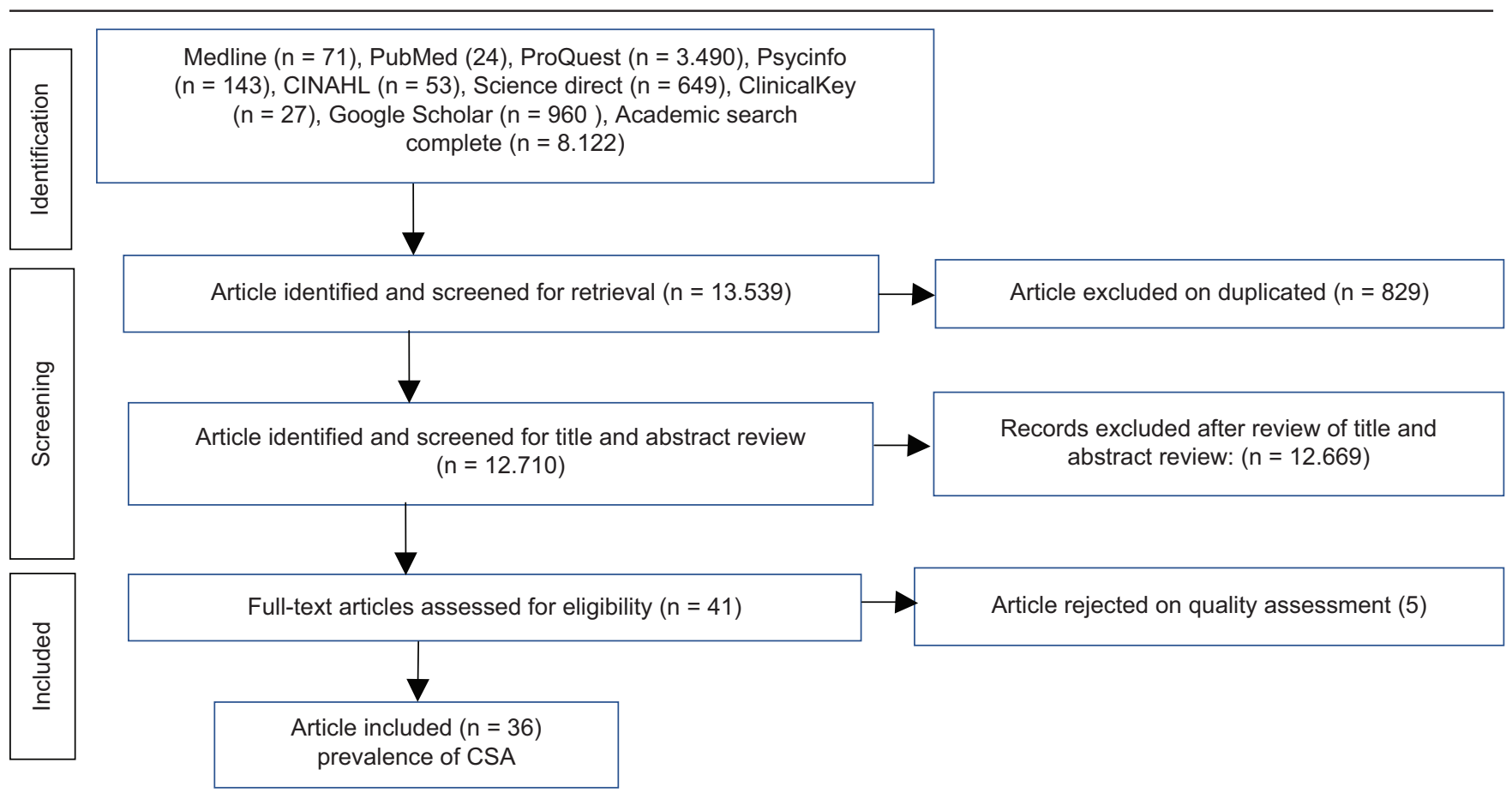

Figure 1: The search strategy based on preferred reporting items for systematic reviews and meta-analyzes flow diagram

eligibility. Every author filled out a study data extraction form for an article that qualified. Those articles were chosen to report the results of the latest literature about the prevalence of CSA. Peer review articles identified from electronic databases were selected and stored in the EndNote X5.01 application. To reduce the risk of bias in this review every effort was made by a panel of experts consisting of three trained academics.

\section{Quality appraisal process}

Study quality in the literature was assessed using the Joanna Briggs Institute (JBI) Critical Appraisal Checklist tool for a prevalence study [37]. As for the mixed method and qualitative study, the study synthesis was carried out using an interpretive perspective in which the identified themes were then combined through a list of descriptive themes [38].

\section{Data extraction and analysis}

Data extraction included: 1) author; 2) year of publication; 3) the geographical area of the study; 4) study design; 5) sample characteristics (age, sex, sample size); 6) age at first exposure to CSA; 7) CSA types assessed according to WHO classification [39], namely non-contact / non-specific CSA (CSA that does not involve physical contact or aspects of unclear physical contact, e.g., exhibitionism, obscene exposure, voyeurism), CSA contact (any CSA including physical contact that does not involve penetration, e.g., non-genital fondling, kissing, or genital touch), and penetration (any CSA including physical contact that involves penetration, e.g., anal, oral, or vaginal sex);
7) CSA prevalence based on sex; 8) characteristics of CSA perpetrator and relationship to the victims; 9) impact of CSA; and 10) place of offence.

\section{Results}

\section{Characteristics of Eligible Studies}

This systematic review summarized what was known about CSA and the status of research on CSA in Asia over the past decade. The articles selected described mostly quantitative research (26 studies), with 1 mixed method study, and 9 qualitative studies (Tables 1, 2 and 3). 24 studies used a cross-sectional design, while two studies used a retrospective design. Qualitative studies were also identified in several studies, including one mix-methods study, seven phenomenological studies, one ethnography study, and a grounded design study.

There was a big difference in sample size. Sample size varied in general population-based studies from 51-18.34 for quantitative-mixed methods and 1-151 for qualitative design (children and adults). Most the studies (25/36) included both male and female samples, while several other studies (6/36) were women only and (5/36) were men only. In 27 quantitative-mixed methods research studies, most of the research (16/27) was carried out on general samples and a small part was carried out on victims $(6 / 27)$, students $(1 / 27)$, college students (1/27), MSM (2/27), and juvenile prisoners (1/27). In a qualitative study, most of studies was carried out on sample victims with certain populations such as 
AQ5 Table 1: The prevalence of CSA on quantitative, mixed methods, and qualitative according to study characteristic

\begin{tabular}{|c|c|c|c|c|c|c|c|c|}
\hline & Author, Country, setting & $\begin{array}{l}\text { Period of } \\
\text { prevalence }\end{array}$ & Study design & Sample size, age (year) & $\begin{array}{l}\text { Ages of first } \\
\text { exposure } \\
\text { (years) }\end{array}$ & Impact & Perpetrators (\%) & $\begin{array}{l}\text { Places of offence } \\
(\%)\end{array}$ \\
\hline \multirow[t]{5}{*}{ AQ6 } & $\begin{array}{l}\text { Xu et al., } 2017 . \\
\text { China. (Community) }\end{array}$ & Childhood & Cross-sectional & $\begin{array}{l}2.050 \text { MSM: } 1,030 \\
\text { noncontact (mean age } \\
25.15) .1,020 \text { contact, } \\
\text { (mean age } 25.05 \text {-years) }\end{array}$ & $\mathrm{X}$ & $\begin{array}{l}\text { High risk HIV, } \\
\text { high risk to be in } \\
\text { a relationship with } \\
\text { a man }\end{array}$ & $\mathrm{X}$ & $\mathrm{X}$ \\
\hline & $\begin{array}{l}\text { Lin et al., } 2011 \\
\text { China (Community) }\end{array}$ & Childhood & Cross-sectional & $\begin{array}{l}683 \text { rural children and } \\
\text { adolescents }(8-18)\end{array}$ & $<16$ years & $\begin{array}{l}\text { Non-contact CSA: } \\
\text { Suicidal ideation, } \\
\text { suicide attempt } \\
\text { Contact CSA: } \\
\text { smoking, cigarette } \\
\text { drinking, alcohol } \\
\text { binge, suicide } \\
\text { attempt, suicidal } \\
\text { ideation }\end{array}$ & $\begin{array}{l}\text { Men experienced at least } \\
\text { one of the nine types of } \\
\text { CSA (13.5). Experienced } \\
\text { verbal harassment/sexual } \\
\text { joke/were exposed to } \\
\text { sexually explicit materials } \\
\text { (97) }\end{array}$ & $\begin{array}{l}\text { The prevalence of } \\
\text { CSA was higher } \\
\text { in non-boarding } \\
\text { children compared } \\
\text { to boarding } \\
\text { children }\end{array}$ \\
\hline & $\begin{array}{l}\text { Chan and Khodabakhsh } 2013 . \\
\text { China. (School) }\end{array}$ & Childhood & Cross-sectional & $\begin{array}{l}18.341 \text { students in } \\
\text { grades } 9-12 .(15-17, \\
\text { mean age } 15.86)\end{array}$ & $x$ & $\mathrm{x}$ & $\begin{array}{l}\text { Perpetrators by known } \\
\text { adult (3.8), peer ( } 2.6)\end{array}$ & $x$ \\
\hline & $\begin{array}{l}\text { Han et al. 2011. South Korea. } \\
\text { (Community) }\end{array}$ & Childhood & Study retrospective & $\begin{array}{l}1,043 \text { adult males, } \\
(19-54 \text { years })\end{array}$ & $13-17$ years & $x$ & $\begin{array}{l}\text { Family members, } \\
\text { acquaintances, relatives, } \\
\text { neighbors, friends }\end{array}$ & $x$ \\
\hline & $\begin{array}{l}\text { Zhang et al. } 2016 . \\
\text { China. (The juvenile correction } \\
\text { institution) }\end{array}$ & Childhood & Cross-sectional, & $\begin{array}{l}358 \text { male juvenile } \\
\text { prisoners. } \\
(15-18) .\end{array}$ & $x$ & $\begin{array}{l}\text { Psychological } \\
\text { distress }\end{array}$ & $x$ & $x$ \\
\hline \multirow[t]{12}{*}{ AQ6 } & $\begin{array}{l}\text { Xu et al. } 2018 \\
\text { China. (Community) }\end{array}$ & Childhood & Cross-sectional & $\begin{array}{l}999 \text { men } \\
(\geq 18, \text { mean age; } 25.06) .\end{array}$ & $x$ & $\begin{array}{l}\text { Psychological } \\
\text { distress, risk } \\
\text { sexual behaviors, } \\
\text { substance use, HIVI } \\
\text { STIs risks }\end{array}$ & $x$ & $x$ \\
\hline & $\begin{array}{l}\text { Wahab et al. } 2013 \\
\text { Malaysia. (Community) }\end{array}$ & Childhood & Cross-sectional & $\begin{array}{l}51 \text { young sexually } \\
\text { abused female }(12-20) \text {. }\end{array}$ & $\begin{array}{l}\text { Mean age: } 14.1 \\
\pm 2.19 \text { years }\end{array}$ & Depression & $\begin{array}{l}\text { Acquaintances }(78,43) \text {, } \\
\text { stranger }(21,57)\end{array}$ & $\mathrm{x}$ \\
\hline & $\begin{array}{l}\text { Kumar et al. } 2019 \\
\text { India. (School) }\end{array}$ & Childhood & Cross-sectional & $\begin{array}{l}\text { 6.957 Adolescents } \\
\text { classes } 8-10 . \\
(13-16) .\end{array}$ & $\mathrm{x}$ & $x$ & $\begin{array}{l}\text { Peer (adults, children, } \\
\text { adolescents) }\end{array}$ & $\begin{array}{l}\text { off home, } \\
\text { phone, bus, sex } \\
\text { books, }\end{array}$ \\
\hline & $\begin{array}{l}\text { Tomori et al., } 2016 . \\
\text { India. (Community) }\end{array}$ & Childhood & Mixed-methods & $\begin{array}{l}11,788 \text { adult MSM. } \\
\text { (Median age }=25, \\
\text { quantitative) } .363 \mathrm{MSM} \\
\text { (median age }=30, \\
\text { qualitative) }\end{array}$ & $x$ & High risk HIV & $\mathrm{x}$ & $x$ \\
\hline & $\begin{array}{l}\text { Tang et al., } 2018 . \\
\text { China. (Colleges) }\end{array}$ & Childhood & Cross-sectional & $\begin{array}{l}17,966 \text { college students. } \\
(18-25) \text {. }\end{array}$ & $\leq 14$ years & $\begin{array}{l}\text { Adverse reproductive } \\
\text { health, high risky } \\
\text { sexual behaviors, }\end{array}$ & $\begin{array}{l}\text { Acquaintances/ friends } \\
\text { (34.6), intimate partners } \\
(24.7)\end{array}$ & $\mathrm{x}$ \\
\hline & $\begin{array}{l}\text { Wei and Chen.2012. } \\
\text { Taiwan. (School) }\end{array}$ & Childhood & Cross-sectional & $\begin{array}{l}\text { 1,376 Adolescents, } \\
\text { classes 7-9. }\end{array}$ & $x$ & $\mathrm{x}$ & Peer & School \\
\hline & $\begin{array}{l}\text { Nan Li et al., } 2015 . \\
\text { Hanoi, Shanghai, and Taipei. } \\
\text { (Community) }\end{array}$ & Childhood & Cross-sectional, & 16.257 people (15-24). & $\begin{array}{l}\text { Mean age: } 10,5 \\
\text { years }\end{array}$ & $x$ & $\begin{array}{l}\text { Relatives }(24) \text {, } \\
\text { supervisors/ teachers } \\
(1,6) .\end{array}$ & $x$ \\
\hline & $\begin{array}{l}\text { Sumner et al. } 2016 . \\
\text { Cambodia. } \\
\text { (Community) }\end{array}$ & Childhood & Cross-sectional & 1255 males $(13-24)$. & $\leq 10$ years & $\begin{array}{l}\text { Penile discharge, } \\
\text { suicidal ideation, } \\
\text { depression, anxiety }\end{array}$ & $\begin{array}{l}\text { Friends/ acquaintance/ } \\
\text { neighbor (54.2), relative } \\
(37.0), \text { family member/ } \\
\text { other (4.7), romantic } \\
\text { partner (4.0), stranger }(0)\end{array}$ & $\begin{array}{l}\text { Victim's } \\
\text { home (41.9), } \\
\text { perpetrator's } \\
\text { home: (6.1), } \\
\text { residence/ } \\
\text { business (11.6), } \\
\text { outdoors (21.8), } \\
\text { school (11.7), } \\
\text { public event/ party } \\
\text { (4.3), other (2.7). }\end{array}$ \\
\hline & $\begin{array}{l}\text { Chandrasiri et al. 2017. Sri } \\
\text { Lanka. (Community) }\end{array}$ & Childhood & Cross-sectional & $\begin{array}{l}132 \text { victims. }(<16 \\
\text { Mean=13.9 years })\end{array}$ & $\begin{array}{l}<16 \text { years. } \\
68.9 \text { had } \\
\text { attained } \\
\text { menarche at } \\
\text { the time of } \\
\text { CSA. (pre- } \\
\text { pubertal age) }\end{array}$ & $\begin{array}{l}\text { Adverse } \\
\text { psychological }\end{array}$ & $\begin{array}{l}\text { The perpetrator was } \\
\text { known ( } 94) \text { : } \\
\text { Boyfriend ( } 28.8) \text {, father/ } \\
\text { step father/ grandparent } \\
\text { (10.6), other relative } \\
(5.3), \text { known non-relative } \\
\text { (46.2), stranger (5.3) Age } \\
\text { of the perpetrator =11 - } \\
65 \text { years old }\end{array}$ & $\begin{array}{l}\text { Offender's habitat } \\
\text { (55.3), Victim's } \\
\text { home (23.5), } \\
\text { Secluded areas } \\
(11.4)\end{array}$ \\
\hline & $\begin{array}{l}\text { Karayianni et al. 2017. Cyprus. } \\
\text { (School, universities, youth } \\
\text { organizations) }\end{array}$ & Childhood & Cross-sectional & $\begin{array}{l}1.852 \text { adolescents-young } \\
\text { adults. (15-25). }\end{array}$ & $x$ & $\begin{array}{l}\text { Violence exposure, } \\
\text { psychological } \\
\text { victimization, } \\
\text { neglect, physical } \\
\text { punishment }\end{array}$ & $\begin{array}{l}\text { Stranger ( } 36) \text {, friends/ } \\
\text { acquaintance (32) } \\
\text { relative (8) }\end{array}$ & $\mathrm{x}$ \\
\hline & $\begin{array}{l}\text { Bae et al. } 2017 . \\
\text { South Korea. } \\
\text { (Children's Center for sexual } \\
\text { abuse) }\end{array}$ & Childhood & Cross-sectional, & 162 children. (3-18). & $x$ & $x$ & $\begin{array}{l}\text { Male }(96.9) \text {. Age: } \\
10-19 \text { years }(35,2) \text {. } \\
\text { Acquaintance: family- } \\
\text { relative and other }(61,1) \text {, } \\
\text { Stranger }(38,9)\end{array}$ & $\mathrm{x}$ \\
\hline & $\begin{array}{l}\text { Sumampouw et al. } 2020 . \\
\text { Indonesia (Community) }\end{array}$ & Childhood & Cross-sectional & $\begin{array}{l}179 \text { police files of CSA } \\
\text { case. }(<18, \text { mean } \\
\text { age }=12.4)\end{array}$ & $x$ & $\begin{array}{l}\text { Pregnancy, pain in } \\
\text { genitalia, }\end{array}$ & $x$ & $x$ \\
\hline
\end{tabular}


Table 1: (Continued)

\begin{tabular}{|c|c|c|c|c|c|c|c|}
\hline Author, Country, setting & $\begin{array}{l}\text { Period of } \\
\text { prevalence }\end{array}$ & Study design & Sample size, age (year) & $\begin{array}{l}\text { Ages of first } \\
\text { exposure } \\
\text { (years) }\end{array}$ & Impact & Perpetrators (\%) & $\begin{array}{l}\text { Places of offence } \\
\text { (\%) }\end{array}$ \\
\hline $\begin{array}{l}\text { Al-Mahroos and Al-Amer. } 2011 . \\
\text { Bahrain. (Community) }\end{array}$ & Childhood & Retrospective & $\begin{array}{l}440 \text { children victims. (9 } \\
\text { months-17, mean age } \\
=8) .\end{array}$ & $\mathrm{X}$ & $\begin{array}{l}\text { Gonorrhea, non- } \\
\text { specific erythema, } \\
\text { encopresis, hymnal } \\
\text { rupture, abrasions/ } \\
\text { lacerations, anal tear, } \\
\text { bleeding, anal laxity, } \\
\text { abnormal discharge, } \\
\text { over-sexualized } \\
\text { behaviors, } \\
\text { pregnancy }\end{array}$ & $\mathrm{x}$ & $\begin{array}{l}\text { Houses (8), } \\
\text { neighbors' homes } \\
\text { (7), schools } \\
\text { (6), shops (6), } \\
\text { mosques (2), } \\
\text { abuser homes (1), } \\
\text { unknown places } \\
\text { (7). }\end{array}$ \\
\hline $\begin{array}{l}\text { Debt and Walsh.2012 } \\
\text { India. (School) }\end{array}$ & Childhood & Cross-sectional & $\begin{array}{l}160 \text { boys } \& 160 \text { girls } \\
(14-19) .\end{array}$ & $\mathrm{x}$ & $\begin{array}{l}\text { Poor social } \\
\text { adjustment }\end{array}$ & $\mathrm{x}$ & $\mathrm{x}$ \\
\hline $\begin{array}{l}\text { Choi et al. } 2015 . \\
\text { South Korea. } \\
\text { (Clinic/region } \\
\text { al victim assistance center for } \\
\text { CSA) }\end{array}$ & Childhood & Cross-sectional & $\begin{array}{l}92 \text { victims. } \\
\text { (<13 years, mean: } 9.07)\end{array}$ & $\mathrm{x}$ & $x$ & $\begin{array}{l}\text { Male (93.5). Age mean: } \\
27.56 \text { years. Immediate } \\
\text { family: } 5 \text { Father, } 1 \text { brother/ } \\
\text { sister. ( } 6.9 \text { ) Extended } \\
\text { family: } 7 \text { cousin, } 4 \\
\text { stepfather, } 2 \text { uncle, } 1 \\
\text { grandfather, } 1 \text { stepbrother } \\
\text { (17.2) Informal guardian: } \\
5 \text { neighbor, } 4 \text { parent's } \\
\text { friend, } 4 \text { teacher, } 4 \\
\text { service providers, } 1 \\
\text { mother's boyfriend (19.5) } \\
\text { Peer group: } 8 \text { friend, } 5 \\
\text { upper-class students, } 5 \\
\text { boyfriends (20.7) Stranger } \\
\text { (21.8), Unknown (13.8) }\end{array}$ & $\begin{array}{l}\text { Victim's } \\
\text { house: (30.4), } \\
\text { perpetrator's } \\
\text { house/ automobile } \\
\text { (23.9), public } \\
\text { places (32.6), } \\
\text { Unknown (13) } \\
\end{array}$ \\
\hline $\begin{array}{l}\text { Pereira et al. } 2020 . \\
\text { Cambodia. (Community) }\end{array}$ & Childhood & Cross-sectional & 586 children. (13-17). & $\mathrm{x}$ & $\mathrm{x}$ & $\mathrm{x}$ & $x$ \\
\hline $\begin{array}{l}\text { Chan et al. } 2011 \\
\text { Hong Kong, China. } \\
\text { (Community) }\end{array}$ & Childhood & Cross-sectional, & 5,049 Chinese. $(>16)$. & $x$ & $\begin{array}{l}\text { IPV sexual, suicidal } \\
\text { ideation, } \\
\text { IPV physical }\end{array}$ & $\begin{array}{l}\text { Relatives/friends (51.2), } \\
\text { strangers }(34.9), \\
\text { core family members } \\
(14.0)\end{array}$ & $\mathrm{x}$ \\
\hline $\begin{array}{l}\text { L. F. Chan. } 2013 . \\
\text { Malaysia. (Community) }\end{array}$ & Childhood & Cross-sectional & $\begin{array}{l}4.581 \text { adolescents. } \\
(17-18) \text {. }\end{array}$ & $\mathrm{x}$ & $\begin{array}{l}\text { Suicidal plans, } \\
\text { suicidal ideation, } \\
\text { deliberate self-harm }\end{array}$ & $x$ & $\mathrm{x}$ \\
\hline $\begin{array}{l}\text { Lam. 2014. Hong Kong, China. } \\
\text { (Community and clinic) }\end{array}$ & Childhood & Cross-sectional & $\begin{array}{l}800 \text { (community) }+30 \\
\text { (clinic) adolescent. (13 } \\
-16) .\end{array}$ & $\begin{array}{l}\text { Mean age } \\
12.2 \text { years } \\
\text { (respondent in } \\
\text { community). } \\
\text { Mean age } \\
12.41 \text { years } \\
\text { (respondent in } \\
\text { clinic) }\end{array}$ & $\begin{array}{l}\text { Threat and the pain } \\
\text { associated with the } \\
\text { CSA's experience }\end{array}$ & $\begin{array}{l}\text { Intrafamilial respondent in } \\
\text { clinic (30), } \\
\text { Intrafamilial respondent } \\
\text { in community: parent, } \\
\text { stepparent, grandparent, } \\
\text { sibling, other relative (12). } \\
\text { Majority: male abusers }\end{array}$ & $\begin{array}{l}\text { Home's victims: } \\
\text { respondent in } \\
\text { clinic (30) }\end{array}$ \\
\hline $\begin{array}{l}\text { Choi and Oh. } 2013 \text {. } \\
\text { Korea (Public counseling center } \\
\text { for sexual abuse) }\end{array}$ & Childhood & Cross-sectional & 495 children. (4-13). & $\begin{array}{l}\text { Median age: } \\
7.64\end{array}$ & $\begin{array}{l}\text { PTSD, disorder, } \\
\text { depressive disorder, } \\
\text { anxiety, disruptive } \\
\text { behavior disorders, } \\
\text { eating disorder, tic } \\
\text { disorder, enuresis, }\end{array}$ & $\begin{array}{l}\text { Stranger ( } 41.7) \text {, } \\
\text { nonfamily acquaintance: } \\
(37.8) \text {, relative }(13.3) \text {, } \\
\text { immediate family }(7.2)\end{array}$ & $\mathrm{x}$ \\
\hline $\begin{array}{l}\text { Wang et al. } 2016 . \\
\text { Southern Taiwan }\end{array}$ & Childhood & Cross-sectional & 55 CSA cases. & $\begin{array}{l}\text { Mean age } \\
5.71 \pm 3.00 \\
\text { years }\end{array}$ & PTSD & $\mathrm{x}$ & $\mathrm{x}$ \\
\hline $\begin{array}{l}\text { Bae et al. } \\
2018 . \\
\text { South Korea. } \\
\text { (Children's and adolescents } \\
\text { Center for sexual abuse) }\end{array}$ & Childhood & Cross-sectional & 63 victims. (8-16). & $\mathrm{x}$ & PTSD & $\begin{array}{l}\text { Acquaintances }(79,4) \text {, } \\
\text { stranger }(20,6)\end{array}$ & $\mathrm{x}$ \\
\hline $\begin{array}{l}\text { Wang and Heppner. } \\
\text { 2011. Taiwan. } \\
\text { (Community) }\end{array}$ & Childhood & Grounded qualitative & $\begin{array}{l}10 \text { female Taiwanese } \\
\text { victims. }(20-39)\end{array}$ & $5-9$ years & $\begin{array}{l}\text { Post-abuse stress, } \\
\text { disrupted victims trust } \\
\text { toward others, felt } \\
\text { victims' bodies were } \\
\text { physically damaged, } \\
\text { discomfort interacted, } \\
\text { fear, ashamed/ } \\
\text { angry with victim's } \\
\text { self, anger toward } \\
\text { others (especially } \\
\text { men), worried } \\
\text { of shunned by } \\
\text { marriage associated } \\
\text { loss virginity and } \\
\text { chastity, mistrust, } \\
\text { suicidal ideation, } \\
\text { concentration } \\
\text { difficulties, CSA } \\
\text { events flashbacks of } \\
\text { intrusive-distressing, } \\
\text { psychosomatic } \\
\text { symptoms, feelings } \\
\text { of isolation, feelings } \\
\text { of helplessness, } \\
\text { hopelessness,. }\end{array}$ & $\begin{array}{l}\text { Father, cousins, brother, } \\
\text { uncle, ex-boyfriend, } \\
\text { security guard at } \\
\text { elementary school, } \\
\text { neighbor, renovator of } \\
\text { victim's house. }\end{array}$ & $x$ \\
\hline
\end{tabular}


Table 1: (Continued)

\begin{tabular}{|c|c|c|c|c|c|c|c|}
\hline Author, Country, setting & $\begin{array}{l}\text { Period of } \\
\text { prevalence }\end{array}$ & Study design & Sample size, age (year) & $\begin{array}{l}\text { Ages of first } \\
\text { exposure } \\
\text { (years) }\end{array}$ & Impact & Perpetrators (\%) & $\begin{array}{l}\text { Places of offence } \\
(\%)\end{array}$ \\
\hline $\begin{array}{l}\text { Batool and Abtahi. } \\
\text { 2017. Pakistan (Community) }\end{array}$ & Childhood & $\begin{array}{l}\text { Phenomenological } \\
\text { qualitative }\end{array}$ & $\begin{array}{l}8 \text { adolescents of CSA } \\
\text { victims ( } 5 \text { boys and } 3 \\
\text { girls). (14-17) }\end{array}$ & $\mathrm{X}$ & $\begin{array}{l}\text { Poor impact on } \\
\text { psychological/ } \\
\text { learning/ future } \\
\text { goals, very rigid, lost } \\
\text { victims' respect and } \\
\text { dignity, distraction } \\
\text { of social interaction, } \\
\text { low confidence, } \\
\text { negative self-image, } \\
\text { victims could } \\
\text { not cherish their } \\
\text { childhood }\end{array}$ & $\mathrm{x}$ & $\mathrm{x}$ \\
\hline $\begin{array}{l}\text { Wijngaarden et al. } \\
\text { 2013.Pakistan (Community) }\end{array}$ & Childhood & $\begin{array}{l}\text { Phenomenological } \\
\text { qualitative }\end{array}$ & $\begin{array}{l}10 \text { young feminized men } \\
\text { of CSA victims. }(14-20)\end{array}$ & $\begin{array}{l}\text { average age : } \\
11,2 \text { years old }\end{array}$ & $\begin{array}{l}\text { Anal STI, high risk of } \\
\text { STI and HIV. }\end{array}$ & $\begin{array}{l}\text { Customer/ stranger, } \\
\text { friends, uncle, older } \\
\text { brother, neighbor, } \\
\text { policeman }\end{array}$ & $\mathrm{x}$ \\
\hline $\begin{array}{l}\text { Chouliara and Narang. } \\
\text { 2017. India. (community) }\end{array}$ & Childhood & $\begin{array}{l}\text { Phenomenological } \\
\text { qualitative }\end{array}$ & $\begin{array}{l}20 \text { adult survivors. } \\
(6 \text { males, } 14 \text { females; } \\
\text { age: } 24-54 \text { ) }\end{array}$ & $\mathrm{x}$ & $\begin{array}{l}\text { Frigidity, } \\
\text { psychological, } \\
\text { shame, blame, } \\
\text { stigma, guilt, } \\
\text { major depression, } \\
\text { traumatic, low self- } \\
\text { worth. Homosexual, } \\
\text { adverse physical } \\
\text { health. }\end{array}$ & $\mathrm{x}$ & $\mathrm{x}$ \\
\hline $\begin{array}{l}\text { Kaiser and Sinanan. } 2020 . \\
\text { Bangladesh } \\
\text { (community) }\end{array}$ & Childhood & $\begin{array}{l}\text { Phenomenological } \\
\text { qualitative }\end{array}$ & $\begin{array}{l}12 \text { female street children. } \\
(13-14 \text { y) }\end{array}$ & $x$ & $\begin{array}{l}\text { Fear, worthless felt } \\
\text { horrific emotional } \\
\text { toll on the children, } \\
\text { become to sex } \\
\text { workers }\end{array}$ & $\begin{array}{l}\text { Uncles' Victim, } \\
\text { stepmother's brother, }\end{array}$ & Street \\
\hline $\begin{array}{l}\text { Senaratna. } \\
\text { 2015. Srilanka. (Community) }\end{array}$ & Childhood & $\begin{array}{l}\text { Phenomenological } \\
\text { qualitative }\end{array}$ & $\begin{array}{l}151 \text { participants. (> 18) } \\
\text { participants for FGD } \\
\text { (School teachers, } \\
\text { community youth groups } \\
\text { \& members of civil } \\
\text { organizations), } \\
30 \text { participants for SSIs } \\
\text { (Community leaders, } \\
\text { religious leaders, } \\
\text { social workers, primary } \\
\text { healthcare workers) }\end{array}$ & $\mathrm{x}$ & $\begin{array}{l}\text { psychological } \\
\text { trauma, physical } \\
\text { trauma, pregnancies, } \\
\text { infections, negative } \\
\text { societal attitudes, } \\
\text { negative social } \\
\text { stigma, humiliation } \\
\text { within family/ } \\
\text { community/ } \\
\text { neighborhood/ } \\
\text { school }\end{array}$ & $\begin{array}{l}\text { Fathers, brothers, } \\
\text { grandfathers, uncles, } \\
\text { cousins, neighbours, } \\
\text { friends, boy-friends, } \\
\text { unknown persons }\end{array}$ & $\begin{array}{l}\text { Home, } \\
\text { neighborhood, } \\
\text { school, lodges, } \\
\text { hotel rooms }\end{array}$ \\
\hline $\begin{array}{l}\text { Eisenbruch. } 2019 \\
\text { Cambodia. (Community) }\end{array}$ & Childhood & $\begin{array}{l}\text { Person-centered } \\
\text { ethnography. }\end{array}$ & $\begin{array}{l}61 \text { victims: } 50 \text { girls and } \\
11 \text { boys. }(2-18) \text {. } \\
\text { Informant: } 39 \text { mothers, } \\
20 \text { fathers, } 16 \\
\text { grandparents, } 2 \text { elder } \\
\text { sisters, } 1 \text { aunt, and } 8 \\
\text { other relatives of abused } \\
\text { Children, } 6 \text { perpetrators } \\
\text { and their families }\end{array}$ & $\mathrm{x}$ & $\mathrm{x}$ & $\begin{array}{l}\text { Father, stepfather, other } \\
\text { close relative/ uncle ( } 13 \\
\text { people), neighbor ( } 3 \\
\text { people), lay Buddhist } \\
\text { officiant ( } 15 \text { people), } \\
\text { monk ( } 21 \text { people) }\end{array}$ & $\mathrm{x}$ \\
\hline $\begin{array}{l}\text { Mulya. } 2018 \\
\text { Indonesia. (Community) }\end{array}$ & Childhood & $\begin{array}{l}\text { Phenomenological } \\
\text { qualitative }\end{array}$ & $\begin{array}{l}3 \text { young Indonesians } \\
\text { who have engaged } \\
\text { in sexual interaction } \\
\text { with adults when they } \\
\text { were children: } 1 \text { college } \\
\text { student, } 1 \text { high school } \\
\text { student, } 1 \text { office worker }\end{array}$ & $5-12$ years & $\begin{array}{l}\text { Feel ashamed, hate/ } \\
\text { afraid of men, sex, \& } \\
\text { marriage }\end{array}$ & $\begin{array}{l}\text { Acquaintances (friend of } \\
\text { uncle, female cousins, } \\
\text { parents' employee, } \\
\text { friends), stranger }\end{array}$ & $\begin{array}{l}\text { Victim's home, } \\
\text { perpetrator's } \\
\text { neighborhood, } \\
\text { church }\end{array}$ \\
\hline $\begin{array}{l}\text { Chan and Khodabakhsh. } 2020 \\
\text { Malaysia (community) }\end{array}$ & Childhood & $\begin{array}{l}\text { Phenomenological } \\
\text { qualitative }\end{array}$ & One victim. (22) & $\mathrm{x}$ & $\begin{array}{l}\text { Negative body } \\
\text { image, fear of } \\
\text { interpersonal } \\
\text { relationships, } \\
\text { emotional distortion, } \\
\text { self-thought } \\
\text { distortion, }\end{array}$ & Step father & $\mathrm{x}$ \\
\hline
\end{tabular}

migrant children, street girls, street boys, feminist men (8/9), and non-victims (1/9).

\section{Discussion}

A systematic review approach was conducted with an overview of studies. We identified 36 studies that met the inclusion criteria for estimating CSA in
Asian countries in papers published between January 2011 and November 2020. This review highlights different study populations (children, adolescents, or adults; vulnerable populations) and different study settings (school, college, or community-based) adding to the practical and statistical challenges of presenting pooled prevalence estimates.

This review summarizes previously known CSA victimization characteristics and the current status of CSA research in countries in Asia. This review added little knowledge about CSA and paid attention to the 
extent and severity of the current epidemic in Asia. Our review of 36 recent original studies on CSA shows that CSA is highly prevalent in Asia. This is consistent with previous research [5], [12], [48] reported that girls have a higher level of susceptibility than boys to CSA. The estimated prevalence range of CSA is $2.2 \%-94 \%$ among girls and 1.7 to $49.5 \%$ among boys, which is higher than the global estimate of $2 \%-56 \%$ for girls and $0.4 \%-44 \%$ for boys [49].

Girls have a higher risk than boys at being victims of CSA. Similar sex differences were reported in previous reviews for estimates of overall prevalence [33]. This difference in estimates of male and female CSA may be due to methodological problems, where the samples from the study used vary; some use general samples, MSM samples, or victims CSA samples. The prevalence of CSA in boys lower than that of girls was possibly due to underreported cases or a lack of data on the prevalence of CSA among MSM [28], [50]. The prevalence rate was much higher among MSM, rural children, and college students. Furthermore, this study used various sampling strategies, various operational definitions of CSA, several study populations (children, adolescents, or adults; vulnerable populations), and different study settings (at school, college, or community-based). This study adds benefits to the practical and methodological statistics from presenting collected prevalence estimates, comparisons between studies, and cross-population comparisons. Our review found that studies with a comprehensive definition of CSA (contact, non-contact, and penetrative) reported a higher prevalence of CSA. CSA incident reporting was required. This is compared with the previous review literature research conducted by Selengia et al. (2020). The prevalence of CSA in Asia was found to be higher than in previous studies [51].

Our findings showed that it was important to differentiate between types of CSA to obtain a more adequate estimate. We found the highest CSA prevalence estimates for penetrative type CSA abuse $(0.5 \%-88.24 \%$ of girls and $1.7 \%-57.1 \%$ of boys $)$ higher than the international estimate of $15.1 \%(95 \%$ $\mathrm{Cl}: 12.9$ - 17.7) for women and 6.9\% (5 - 9.5) for men. Slightly lower rates were reported for abuse of the noncontact CSA type (12.6\% - 56.5\% for girls and $0.7 \%$ $68.7 \%$ for boys). The lowest rate for contact CSA (5.3\% - $67.2 \%$ for girls and $2.2 \%-53.3 \%$ for boys) was higher than the international estimate for CSA contacts of $21.2 \%$ (95\% Cl: 17.8 - 25) for women and 10.7\% (6.6 16.8) for men [12]. This was an important finding of this study. The results of this study differ from the results of a meta-analysis study by Barth et al. (2013) in countries around the world regarding the highest for non-contact type CSA and the lowest for forced intercourse type CSA [17].

In this study, it was found that girls have a higher prevalence of CSA compared to boys in all types of CSA; this is in line with Tanaka's (2017) systematic review research in Japan [34]. Characteristics of girls such as shyness, obedience, and fear of stigmatization have an important role in increasing CSA in girls [52]. The lower prevalence of CSA in boys than in girls is possible due to underreporting and the absence of data on CSA prevalence among MSM [28], [50]. The prevalence rate is much higher among MSM, rural children, and college students. There is a need for CSA incident reporting. Under-reporting is associated with frequent disregard for children as individuals with their own rights and often neglects sexual abuse and other forms that children may report [53], [54]. Under-reported CSA can also be linked to gaps in communication between parents and children about CSA issues, guilt, fear of humiliation, rejection from society, and associated socio-cultural stigma, as well as distrust of the government who handle CSA [53], [55], [56], [57], [58]. In addition, the definition of CSA also influences the estimation of the prevalence of CSA. Previous authors have argued that defining CSA in a broad sense (i.e. including noncontact, contact harassment, and forced intercourse in one definition) leads to a higher prevalence estimate compared to using a narrow definition (i.e., only forced sexual intercourse and contact abuse) [39], [59]. This happens because every community has different perceptions about the definition of CSA.

Apart from highlighting the high prevalence, the studies in this review are starting to highlight the impact of CSA. CSA has adverse effects on social, psychological, physical, and health functioning behavior across all ages [60], [61], [62], [63], [64]. All these effects will make the victim feel worthless with himself and believe that sex is something terrible [65], [66] which causes disruption of interactions with the surrounding environment [67] thus reducing their quality of life. CSA has been generally recognized as the reason for much suffering in the lives of the adult survivors [68]. All CSAs tended to have unintended relationships with health conditions and social problems such as acts of victimization through intimate partner violence, becoming perpetrators of CSA in the future, depression, drug abuse, and even suicidal ideation or death [22], [31], [60], [69], [70], [71], [72]. Drug abuse were more likely than heroin subjects to be involved in risky sexual behavior [73]. Some CSA victims dream of a happy future [74] and there are some victims who can perform CSA recovery to eliminate these impacts [75], [76], [77], but it is certainly difficult for victims to move on from what they have experienced. Moreover, Asia has an Eastern culture where chastity is very important for a woman [75]; rape victims are considered dirty women that will never be able to restore their honor [76]. It is sad that this culture is one where they have to maintain the good name of their family by not reporting CSA incidents [75], and that CSA is seen as a bad legacy caused by actions in previous lives [78]. This will increase the chance of CSA perpetrators committing sexual abuse against children as the perpetrators will feel safe from 
Table 2: The mind finding of CSA on quantitative and mixed methods according to study

\begin{tabular}{|c|c|c|c|c|c|c|c|c|}
\hline \multirow[t]{3}{*}{ Author, Country, setting } & \multicolumn{8}{|l|}{ Type of sexual abuse } \\
\hline & \multicolumn{2}{|l|}{ Noncontact (\%) } & \multicolumn{2}{|c|}{ Contact (\%) } & \multicolumn{2}{|c|}{ Penetrating (\%) } & \multicolumn{2}{|c|}{ Prevalence \% } \\
\hline & f & t & क & t & क & t & क & $\frac{p}{t}$ \\
\hline Xu et al., 2017. China. (Community) & $\mathrm{X}$ & $36.2 \%$ & $\mathrm{X}$ & $29.8 \%$ & $\mathrm{X}$ & $\mathrm{X}$ & $\mathrm{X}$ & $15.0-20.4 \%$ \\
\hline Lin et al., 2011 China (Community) & 12.6 & 19.7 & 15.0 & 9.2 & $\mathrm{x}$ & $\mathrm{x}$ & 14.2 & 21.5 \\
\hline Chan et al., 2013. China. (School) & $\begin{array}{l}\text { Verbal sexual }(2,2) \text {, forced } \\
\text { exposure to pornography } \\
(2,4) \text {, } \\
\text { nude photographs } \\
\text { being taken }(2,5) \text {, nude } \\
\text { photographs being } \\
\text { uploaded on the internet } \\
(2,4) \text {. }\end{array}$ & $\begin{array}{l}\text { Verbal sexual }(3,5) \text {, } \\
\text { Forced exposure to } \\
\text { pornography: }(4,4) \text {, Nude } \\
\text { photographs being taken } \\
(1,4), \text { Nude photograph } \\
\text { being uploaded on } \\
\text { Internet }(1,6)\end{array}$ & $\mathrm{x}$ & $\mathrm{x}$ & Rape: 1,9 & Rape:2,5 & 9.3 & 6.6 \\
\hline Han et al. 2011.South Korea. (community) & $x$ & $\begin{array}{l}\text { See their genitalia; }(3.7) \text {, } \\
\text { verbal harassment }(0.7)\end{array}$ & $\mathrm{x}$ & $\begin{array}{l}\text { Forced to engage in } \\
\text { kissing }(2.2) \text {, Asked to } \\
\text { touch the perpetrator's } \\
\text { genitalia ( } 2.7) \text {, }\end{array}$ & $\mathrm{x}$ & 1,7 & $\mathrm{x}$ & $\begin{array}{l}13.5 \% \text { had } \\
\text { experienced at } \\
\text { least one types } \\
\text { of CSA. }\end{array}$ \\
\hline $\begin{array}{l}\text { Zhang et al. 2016. China. (The juvenile } \\
\text { correction institution }\end{array}$ & $x$ & 41 & $\mathrm{x}$ & 53.3 & $\mathrm{x}$ & 5.7 & $x$ & 21.8 \\
\hline Xu et al. 2018. China. (Community) & $\mathrm{x}$ & 11.1 & $\mathrm{x}$ & 29.6 & $\mathrm{x}$ & $\mathrm{x}$ & $\mathrm{x}$ & 40.7 \\
\hline Wahab et al. 2013. Malaysia. (community) & $\mathrm{x}$ & $\mathrm{x}$ & $\mathrm{x}$ & $\mathrm{x}$ & 88.24 & $\mathrm{x}$ & $\mathrm{x}$ & $\mathrm{x}$ \\
\hline Kumar et al. 2019. India. (School) & 11,5 & 68.7 & 9.7 & 18 & 0.5 & 2.7 & 14.0. & 34.2 \\
\hline Tomori et al., 2016. India. (community) & $\mathrm{x}$ & $\mathrm{x}$ & $\mathrm{x}$ & $\mathrm{x}$ & $\mathrm{x}$ & $\mathrm{x}$ & 22.4 & \\
\hline Tang et al., 2018. China. (colleges) & 56.5 & 43.5 & 67.2 & 32.8 & 42.9 & 57.1 & 27.5 & \\
\hline Wei and Chen. 2012. Taiwan. (School) & 20.4 & 26.1 & 5.3 & 8.5 & $\mathrm{x}$ & & 25.4 & \\
\hline $\begin{array}{l}\text { Nan Li et al., 2015. Hanoi, Shanghai, and } \\
\text { Taipei.(community) }\end{array}$ & 0.9 & & 1.0 & & NS & NS & 2.2 & 1.7 \\
\hline $\begin{array}{l}\text { Sumner et al. 2016. Cambodia. } \\
\text { (community) }\end{array}$ & $\mathrm{x}$ & & $\mathrm{x}$ & & $x$ & 3.3 & $\mathrm{x}$ & 5.6 \\
\hline $\begin{array}{l}\text { Chandrasiri et al. 2017.Sri Lanka. } \\
\text { (community) }\end{array}$ & $11.4 \%$ (physical battery), 20 & 5 (libidinous acts) & 39.4( & (intracrural sex) & $\begin{array}{l}\text { Penetrative } \\
\text { digital pen } \\
\text { (4.3), anal }\end{array}$ & $\begin{array}{l}\text { e (61.4), } \\
\text { etration } \\
\operatorname{sex}(10.6)\end{array}$ & 92.4 & 7.6 \\
\hline $\begin{array}{l}\text { Karayianni et al. 2017. Cyprus. (school, } \\
\text { universities, youth organizations) }\end{array}$ & 25.9 & & 7.6 & & 5.8 & & 80 & 21 \\
\hline $\begin{array}{l}\text { Bae et al. 2018.South Korea. } \\
\text { (Children's and adolescents Center for } \\
\text { sexual abuse) }\end{array}$ & $\mathrm{x}$ & & 73.0 & & 27.0 & & 92.1 & 7.9 \\
\hline $\begin{array}{l}\text { Sumampouw et al. } 2020 . \\
\text { Indonesia. (Community) }\end{array}$ & $x$ & & 24.2 & & 75.8 & & 94 & 6 \\
\hline $\begin{array}{l}\text { Al-Mahroos and Al-Amer. 2011. Bahrain. } \\
\text { (community) }\end{array}$ & 11 & & 71.5 & & 61 & & 50.5 & 49.5 \\
\hline Debt \& Walsh.2012. India. (school) & $x$ & & $x$ & & $\mathrm{x}$ & & 25.0 & 11.3 \\
\hline $\begin{array}{l}\text { Choi et al. 2015.South Korea. (Clinic/ } \\
\text { regional victim assistance center for CSA) }\end{array}$ & $\mathrm{x}$ & & $\mathrm{x}$ & & $\mathrm{x}$ & & 77.2 & 22.8 \\
\hline Pereira et al. 2020. Cambodia. (community) & $\mathrm{x}$ & & $\mathrm{x}$ & & $\mathrm{x}$ & & 16.57 & $\mathrm{x}$ \\
\hline $\begin{array}{l}\text { Chan et al. } 2011 \text { Hong Kong, China. } \\
\text { (community) }\end{array}$ & $\mathrm{x}$ & & 0.7 & & 0.2 & & 0.9 & \\
\hline L. F. Chan. 2013.Malaysia. (community) & $\mathrm{X}$ & & $\mathrm{x}$ & & $\mathrm{x}$ & & 21.3 & \\
\hline $\begin{array}{l}\text { Lam. 2014. Hongkong, China. (community } \\
\text { and clinic) }\end{array}$ & 64 (respondent in communit & & $\mathrm{x}$ & & $\begin{array}{l}14 \text { (respon } \\
\text { community } \\
\text { (responder }\end{array}$ & $\begin{array}{l}\text { dent in } \\
\text { nt in clinic) }\end{array}$ & $\begin{array}{l}18.4(r \\
\text { comm }\end{array}$ & ndent in \\
\hline $\begin{array}{l}\text { Choi and Oh. 2013. Korea (Public } \\
\text { counseling center for sexual abuse) }\end{array}$ & $\mathrm{x}$ & & $\mathrm{x}$ & & 33.1 & & 73.1 & \\
\hline Wang et al. 2016. Southern Taiwan & 43.64. Full disclosure (17 ch & iildren), Non full disclosure ( & 7 child & (ren) & $\begin{array}{l}\text { 52.73. Full } \\
\text { disclosure } \\
\text { children). } \\
\text { disclosure } \\
\text { children) }\end{array}$ & $\begin{array}{l}\text { (15 } \\
\text { Non full } \\
(14\end{array}$ & 87.27 & 12.73 \\
\hline $\begin{array}{l}\text { Bae et al. 2017. South Korea. (Children's } \\
\text { Center for sexual abuse) }\end{array}$ & 77.2 & & & & 22.8 & & 90.1 & 9.9 \\
\hline
\end{tabular}

punishment. Meanwhile, our review of the mixed method with the MSM sample provided insight into the influence of CSA history on HIV, where HIV prevalence in the number of MSM experienced CSA was almost twice as high if compared to those without CSA.

In this review, most researchers reported the age of first time the child had CSA at under 12.41 years or the preteen period. The results of this study are different from other studies which reported that the age of first-time children being victims of CSA was less than 12-16 years old [79], [80]. The mean age at risk of experiencing sexual abuse in this study was in the pre-adolescent age for both boys and girls. This is consistent with the results of a study by Karayianni et al. (2017) on 1852 adolescents and young adults in Cyprus which showed that various types of sexual abuse were more likely to occur in adolescents compared to other developmental stages. [81]. The findings in this study are important, considering that many Asian societies still have the perception that young children are unlikely to become victims of sexual abuse.

In the review of this study, it was found that most CSA perpetrators $(37 \%$ - $79.4 \%)$ were people known and close to the victim. Children are easily deceived by offenders who they are comfortable with and consider as friends or protectors. The review is consistent with previous research which reported that CSA perpetrators were the majority of people known to the victims [82], [83], [84], [85]. In fact, the community thinks that it is impossible for people known to the victims to commit sexual crimes against children they know. So far, parents have always reminded their children to be alert to strangers from crimes, including sexual crimes. According to the Crime Victim Center, teaching children 
Table 3: The mind finding of CSA on qualitative according to study

\begin{tabular}{|c|c|}
\hline Author, Country, Setting & Main finding \\
\hline $\begin{array}{l}\text { Wang and Heppner.2011. } \\
\text { Taiwan. (Community) }\end{array}$ & $\begin{array}{l}\text { 1. The recovery of Taiwanese survivors of CSA } \\
\text { is described by the process: (1) Intrapersonal, } \\
\text { interpersonal, sociocultural factors, (2) coping process- } \\
\text { outcomes of CSA survivors } \\
\text { 2. Chastity is important } \\
\text { 3. Lack of knowledge of victims due to their parents } \\
\text { not discussing sexuality with them. They receive is } \\
\text { inadequate. } \\
\text { 4. There is pressure on CSA victims to hide the incidence } \\
\text { because they have to protect their family's good name } \\
\text { from shame }\end{array}$ \\
\hline $\begin{array}{l}\text { Batool and Abtahi. } 2017 . \\
\text { Pakistan. (Community) }\end{array}$ & $\begin{array}{l}\text { CSA victims believe that they will never be able to return } \\
\text { their respect in the eyes of their family and friends if they } \\
\text { find out they are CSA victims. Coping performed by CSA } \\
\text { victims: dissociation, denial, disconnection from offender, } \\
\text { avoiding specific places, distraction, religious beliefs, and } \\
\text { rationalization of the incident of abuse. }\end{array}$ \\
\hline $\begin{array}{l}\text { Wijngaarden et al. } 2013 . \\
\text { Pakistan. (Community) }\end{array}$ & $\begin{array}{l}\text { The experiences of victims it was found that: Young } \\
\text { feminized men face high levels of stigma, violence and } \\
\text { sexual abuse having reportedly been raped during } \\
\text { childhood and early adolescence. Since victims was little, } \\
\text { he acted like a woman and was hated by his parents } \\
\text { because of his behavior }\end{array}$ \\
\hline $\begin{array}{l}\text { Chouliara and Narang. } 2017 . \\
\text { India. (Community) }\end{array}$ & $\begin{array}{l}\text { There were four processes identified in the way victims } \\
\text { described their journey out of CSA, namely: the affected } \\
\text { self-keeping the self together, accurate symbolization, } \\
\text { activation of the recovering self, self-reconnection, } \\
\text { integration, and growth }\end{array}$ \\
\hline $\begin{array}{l}\text { Kaiser and Sinanan. } 2020 . \\
\text { Bangladesh. (Community) }\end{array}$ & $\begin{array}{l}\text { The majority of street children experienced of CSA, } \\
\text { many street children still dream of a happy future, and } \\
\text { the reasons for being street children are poverty, family } \\
\text { violence, and avoiding CSA which is carried out by their } \\
\text { own families }\end{array}$ \\
\hline $\begin{array}{l}\text { Senaratna. 2015. Srilanka. } \\
\text { (Community) }\end{array}$ & $\begin{array}{l}\text { The vulnerability of these migrant girls to abuse, but rarely } \\
\text { disclosed due to social and institutional factors. Girls are } \\
\text { more often sexually abused than boys because girls are } \\
\text { shy and obedient, fearing being stigmatized, }\end{array}$ \\
\hline $\begin{array}{l}\text { Eisenbruch. 2019. Cambodia. } \\
\text { (Community) }\end{array}$ & $\begin{array}{l}\text { CSA is seen as deriving from a "cultural pull," including } \\
\text { a bad legacy caused by actions in a previous life, a bad } \\
\text { character that starts early in life, astrological susceptibility } \\
\text { to abuse, predetermined attachments between children } \\
\text { and abusers, paths to destruction, "and moral blindness } \\
\text { that portrays the perpetrator as innocent. }\end{array}$ \\
\hline $\begin{array}{l}\text { Mulya. 2018. Indonesia. } \\
\text { (Community) }\end{array}$ & $\begin{array}{l}\text { The victim thinks that the meaning of sex is terrible, adults } \\
\text { as powerful but children as helpless and innocent }\end{array}$ \\
\hline $\begin{array}{l}\text { Chan and Khodabakhsh. } \\
\text { 2020. Malaysia. (community) }\end{array}$ & $\begin{array}{l}\text { CSA affects negative perceptions of body image, } \\
\text { difficulties in interpersonal relationships, and distorted } \\
\text { thoughts in CSA victims }\end{array}$ \\
\hline
\end{tabular}

about the dangers of strangers is misleading, let alone not discussing the fact that most children were abused by people they know [86]. Parents have neglected the real danger to their children that comes from individuals close to them. In Asia, parents' perceptions of sexual predators of children should begin to change from thinking that it is impossible for people known to the children to commit acts of sexual assault on their children, including families with blood ties. Parents are their child's first teachers in sexuality education [87] and must provide information to their children about who they should be aware of regarding sexual abuse. This study also highlights the CSA scene. In this study, it was reported that CSA was more common in the perpetrator's house and in the neighborhood. This is possible because the location is quiet and rarely known by other people. The prevalence of CSA can be influenced by place [23]. Thus, parents should supervise their children so that they do not escape the parents' observation where children are not left alone or are able to play in an environment without supervision because crimes occur as a result of opportunities arising.

Important findings in this study indicate that there are many internal and external factors of the victim that contribute to CSA. Most of the causative factors are internal factors of the victim, such as the characteristics of the victim, lack of knowledge, lack of parental protection and supervision of the child, and cultural factors adopted. Street children and children who run away from home are most at risk of experiencing CSA in this study. Even after they grow up and start looking for coping mechanisms, the detrimental effects of CSA can destroy them throughout their lives.

\section{Strengths and limitations}

Strengths in this review were the categorization of CSA into non-contact, contact, and penetrative, the homogeneity of the methodology used, use of an adequate sample, and the fact that all studies were published in peer reviews. However, the analysis was limited by the characteristics of the sample and the age limit of the victim. Retrospective studies can underestimate the prevalence rate because of bias in remembering CSA that occurred in the past, especially if abuse occurred while they studied in preschool years. Sexual abuse of boys committed by female offenders may also not be reported. Therefore, the prevalence rate in this systematic review can be taken into consideration as a minimum rate.

\section{Conclusion}

Based on the results of the review, it can be concluded that CSA is one of the major public health problems in Asia. The majority of victims experience CSA for the first time in their preteen years. CSA perpetrators are mostly people known and close to the victim. Cultural factors have a role in increasing the prevalence of CSA. CSA has adverse physical, psychological, social, and behavioral effects where the victim will suffer a lifetime of trauma, so it is necessary to develop effective CSA prevention interventions involving Asian culture and provide adequate follow-up.

\section{Acknowledgment and Funding}

The authors would like to thank the Library of Universitas Padjadjaran Bandung Indonesia for providing access to databases of my research. We extend our gratitude to the Rector of UNPAD and the Director of Research and Community Service of UNPAD who has funded this research through Padjadjaran University's Doctoral Dissertation Research Grant Scheme. 


\section{Authors' Contribution}

TS designed the study and provided the concept. TS, CEK did screening, collection, and analysis of data. TS, HSM, and $\mathrm{YH}$ wrote the manuscript. TS, HSM, YH, IP and CEK, revised the manuscript for important intellectual contents.

\section{References}

1. Ali M. National policy for children 2013: High on promises, low on budget. Economic and Political Weekly; 2013. p. 22-24.

2. UNICEF. Child Maltreatment, Prevalence, Incidence and Consequences in the East Asia and Pacific Region. New York: UNICEF; 2012

3. Fang X, Fry DA, Brown DS, Mercy JA, Dunne MP, Butchart AR, et al. The burden of child maltreatment in the East Asia and Pacific region. Child Abuse Neglect. 2015;42:146-62. https:// doi.org/10.1016/j.chiabu.2015.02.012

PMid:25757367

4. Behere PB, Mulmule AN. Sexual abuse in 8-year-old child: Where do we stand legally? Indian J Psychol Med. 2013;35(2):203-5. https://doi.org/10.4103/0253-7176.116256

\section{PMid:24049233}

5. Gilbert R, Widom CS, Browne K, Fergusson D, Webb E, Janson $\mathrm{S}$. Burden and consequences of child maltreatment in high-income countries. Lancet. 2009;373(9657):68-81. https:// doi.org/10.1016/s0140-6736(08)61706-7

PMid:19056114

6. Kloppen K, Haugland S, Svedin CG, Mæhle M, Breivik K. Prevalence of child sexual abuse in the Nordic countries: A literature review. J Child Sex Abuse. 2016;25(1):37-55. https:// doi.org/10.1080/10538712.2015.1108944 PMid:26809050

7. Fry D, Blight S. How prevention of violence in childhood builds healthier economies and smarter children in the Asia and Pacific region. BMJ Glob Health. 2016;1(Suppl 2):i3-11. https://doi. org/10.1136/bmjgh-2016-000188

8. Bebbington $\mathrm{PE}$, Jonas $\mathrm{S}$, Brugha $\mathrm{T}$, Meltzer $\mathrm{H}$, Jenkins $\mathrm{R}$, Cooper $\mathrm{C}$, et al. Child sexual abuse reported by an English national sample: Characteristics and demography. Soc Psychiatry Psychiatr Epidemiol. 2011;46(3):255-62. https://doi. org/10.1007/s00127-010-0245-8 PMid:20544176

9. Maniglio R. The impact of child sexual abuse on health: A systematic review of reviews. Clin Psychol Rev. 2009;29(7):647-57. https://doi.org/10.1016/j.cpr.2009.08.003 PMid: 19733950

10. Tang K, Qu X, Li C, Tan S. Childhood sexual abuse, risky sexual behaviors and adverse reproductive health outcomes among Chinese college students. Child Abuse Negl. 2018;84:123-30. https://doi.org/10.1016/j.chiabu.2018.07.038 PMid:30086418

11. Johnson CF. Child sexualabuse. Lancet. 2004;364(9432):462-70. https://doi.org/10.1016/s0140-6736(04)16771-8 PMid:15288746

12. Stoltenborgh M, van IJzendoorn MH, Euser EM, BakermansKranenburg MJ.Aglobal perspective on child sexual abuse: Metaanalysis of prevalence around the world. Child Maltreatment.
2011;16(2):79-101. https://doi.org/10.1177/1077559511403920 PMid:21511741

13. Pinheiro P. World Report on Violence Against Children. Geneva, Switzerland: United Nations Secretary-General's Study on Violence Against Children; 2006.

14. Selmini R. Sexual abuse of children in comparative and international perspective. In: Women and Children as Victims and Offenders: Background, Prevention, Reintegration. Berlin: Springer; 2016. p. 821-55. https://doi. org/10.1007/978-3-319-08398-8_29

15. Pereda N, Guilera G, Forns M, Gómez-Benito J. The international epidemiology of child sexual abuse: A continuation of Finkelhor (1994). Child Abuse Neglect. 2009;33(6):331-42. https://doi.org/10.1016/j.chiabu.2008.07.007 PMid:19477003

16. Choudhry V, Radhika D, Pillai D, Kalokhe AS, Beier K, Patel V. Child sexual abuse in India: A systematic review. PLoS One. 2018;13(10):e0205086. https://doi.org/10.1371/journal. pone.0205086 PMid:30300379

17. Barth J, Bermetz L, Heim E, Trelle S, Tonia T. The current prevalence of child sexual abuse worldwide: A systematic review and meta-analysis. Int J Public Health. 2013;58(3):469-83. https://doi.org/10.1007/s00038-012-0426-1 PMid:23178922

18. Collin-Vézina $D$, Daigneault $I$, Hébert $M$. Lessons learned from child sexual abuse research: Prevalence, outcomes, and preventive strategies. Child Adolesc Psychiatry Ment Health. 2013;7(1):22. https://doi.org/10.1186/1753-2000-7-22 PMid:23866106

19. Cong E, Li Y, Shao C, Chen J, Wu W, Shang X, et al. Childhood sexual abuse and the risk for recurrent major depression in Chinese women. Psychol Med. 2012;42(2):409-17. https://doi. org/10.1017/S0033291711001462 PMid:21835095

20. Miller AB, Schaefer KE, Renshaw KD, Blais RK. PTSD and marital satisfaction in military service members: Examining the simultaneous roles of childhood sexual abuse and combat exposure. Child Abuse Neglect. 2013;37(11):979-85. https://doi org/10.1016/j.chiabu.2013.05.006

PMid:23790507

21. Lacelle C, Hébert M, Lavoie F, Vitaro F, Tremblay RE. Child sexual abuse and women's sexual health: The contribution of CSA severity and exposure to multiple forms of childhood victimization. J Child Sex Abuse. 2012;21(5):571-92. https://doi. org/10.1080/10538712.2012.688932

PMid:22994694

22. Paolucci EO, Genuis ML, Violato C. A meta-analysis of the published research on the effects of child sexual abuse. J Psychol. 2001;135(1):17-36. https://doi.org/10.1080/00223980109603677 PMid:11235837

23. Ji K, Finkelhor D, Dunne M. Child sexual abuse in China: A metaanalysis of 27 studies. Child Abuse Neglect. 2013;37(9):613-22. https://doi.org/10.1016/j.chiabu.2013.03.008

24. UNICEF. Violence against Children in East Asia and the Pacific: A Regional Review and Synthesis of Findings. Bangkok: UNICEF; 2014.

25. Kacker L, Varadan S, Kumar P. Study on Child Abuse: INDIA 2007. Ministry of Women and Child Development, Government of India. Available from: http://www.unodc.org/pdf/india/publications/ htvs_miniweb/childabuse. [Last accessed on 2021 May 25].

26. Chandrasiri MD, Wijewardena DK, Lanerolle SD, Chandrasiri SE, Wijewardena KP, Cooray RT. Child sexual abuse presenting to district general hospital, Chilaw. Ceylon Med J. 2017;62(1):29-33. https://doi.org/10.4038/cmj.v62i1.8430 


\section{PMid:28390329}

27. Dartnall E, Jewkes R. Sexual violence against women: The scope of the problem. Best Pract Res Clin Obstet Gynaecol. 2013;27(1):3-13. https://doi.org/10.1016/j.bpobgyn.2012.08.002 PMid:22940107

28. Tomori C, McFall AM, Srikrishnan AK, Mehta SH, Nimmagadda N, Anand $\mathrm{S}$, et al. The prevalence and impact of childhood sexual abuse on HIV-risk behaviors among men who have sex with men (MSM) in India. BMC Public Health. 2016;16(784):016-3446. https://doi.org/10.1186/s12889-016-3446-6 PMid:27520978

29. Smallbone S, Marshall WL, Wortley R. Preventing Child Sexual Abuse: Evidence, Policy and Practice. Willan; 2013. https://doi. org/10.4324/9781843925606

30. Kumar MT, Kumar S, Singh SP, Kar N. Prevalence of child abuse in school environment in Kerala, India: An ICAST-CI based survey. Child Abuse Negl. 2017;70:356-63. https://doi. org/10.1016/j.chiabu.2017.06.025

\section{PMid:28692832}

31. Lin D, Li X, Fan X, Fang X. Child sexual abuse and its relationship with health risk behaviors among rural children and adolescents in Hunan, China. Child Abuse Neglect. 2011;35(9):680-7. https:// doi.org/10.1016/j.chiabu.2011.05.006

32. Mathews B, Walsh K, Dunne M, Katz I, Arney F, Higgins D, et al. Scoping Study for Research into the Prevalence of Child Abuse in Australia: Prepared for the Royal Commission into Institutional Responses to Child Sexual Abuse [Social Policy Research Centre (SPRC Report 13/16)]; 2016.

33. Pereda N, Guilera G, Forns M, Gómez-Benito J. The prevalence of child sexual abuse in community and student samples: A meta-analysis. Clin Psychol Rev. 2009;29(4):328-38. https:// doi.org/10.1016/j.cpr.2009.02.007

PMid:19371992

34. Tanaka M, Suzuki YE, Aoyama I, Takaoka K, MacMillan HL. Child sexual abuse in Japan: A systematic review and future directions. Child Abuse Neglect. 2017;66:31-40. https://doi. org/10.1016/j.chiabu.2017.02.041

PMid:28291536

35. Hobbs C. The prevalence of child maltreatment in the United Kingdom. Child Abuse Neglect. 2005;29(9):949-51. https://doi.org/10.1016/j.chiabu.2005.08.002 PMid:16159665

36. Moher D, Liberati A, Tetzlaff J, Altman DG. Preferred reporting items for systematic reviews and meta-analyses: The PRISMA statement. Ann Intern Med. 2009;151(4):264-9. https://doi. org/10.7326/0003-4819-151-4-200908180-00135 PMid:19622511

37. Joanna Briggs Institute. The Joanna Briggs Institute Critical Appraisal tools for use in JBI Systematic Reviews: Checklist for Prevalence Studies; 2017.

38. Alaggia R. An ecological analysis of child sexual abuse disclosure: Considerations for child and adolescent mental health. J Can Acad Child Adolesc Psychiatry. 2010;19(1):32-9. https://doi.org/10.1080/16826108.1989.9631438 PMid:20119565

39. Andrews G, Corry J, Slade T, Issakidis C, Swanston H. Child Sexual Abuse Comparative Quantification of Health Risks: Global and Regional Burden of Disease Attributable to Selected Major Risk Factors; 2004. p. 1851-940.

40. Xu W, Zheng L, Zheng Y. Prevalence of non-contact and contact childhood sexual abuse: An internet-based sample of men who have sex with men in China. PLoS One. 2017;12(4):e0175444.

41. Han IY, Lee Y, Yoo SK, Hong J. Prevalence of and risk factors for male sexual abuse: The case of South Korea. J Loss Trauma. 2011;16(1):84-101.
42. Xu W, Zheng L, Song J, Zhang X, Zhang X, Zheng Y. Relationship between childhood sexual abuse and HIV-related risks among men who have sex with men: Findings from mainland China. Arch Sex Behav. 2018;47:1949-57.

43. Tang K, Qu X, Li C, Tan S. Childhood sexual abuse, risky sexual behaviors and adverse reproductive health outcomes among Chinese college students. Child Abuse Negl. 2018;84:123-30.

44. Bae SM, Kang JM, Hwang IC, Cho H, Cho AH. Intelligence is associated with voluntary disclosure in child sexual abuse victims. J Adolesc Health. 2017;61(3):335-41.

45. Choi KS, Choo K, Choi J, Woo YJ. Understanding the dynamics of the victim-perpetrator relationship in child sexual abuse: An examination of the child sex abuse victimization data in South Korea. 2015;10(1):79-97.

46. Choi JY, Oh KJ. The effects of multiple interpersonal traumas on psychological maladjustment of sexually abused children in Korea. J Trauma Stress. 2013;26(1):149-57.

47. Wang LH, Lu T, Tsai MA. Children's disclosure of sexual abuse during early forensic psychiatric evaluation in Southern Taiwan. J Formos Med Assoc. 2016;115(12):1069-75.

48. Radford L, Corral S, Bradley C, Fisher HL. The prevalence and impact of child maltreatment and other types of victimization in the UK: Findings from a population survey of caregivers, children and young people and young adults. Child Abuse Neglect. 2013;37(10);801-13. https://doi.org/10.1016/j.chiabu.2013.02.004 PMid:23522961

49. Radford L, Allnock D, Hynes P. Preventing and Responding to Child Sexual Abuse and Exploitation: Evidence Review. New York: UNICEF; 2015.

50. Wihbey J. Global Prevalence of Child Sexual Abuse. Journalist Resource; 2011. Available from: https://www. journalistsresource.org/studies/global-prevalence-childsexual-abuse. [Last accessed on 2011 Nov 15]. https://doi. org/10.1007/0-306-47200-7_5

51. Selengia V, Thuy HN, Mushi D. Prevalence and patterns of child sexual abuse in selected countries of Asia and Africa: A review of literature. Open J Soc Sci. 2020;8(9):146-60. https://doi. org/10.4236/jss.2020.89010

52. Senaratna C. Sexual abuses of left-behind children of migrant women: Community-perceived vulnerabilities and barriers in prevention. Int J Migration Health Soc Care. 2015;11(4):225-38. https://doi.org/10.1108/ijmhsc-02-2014-0007

53. Carson DK, Foster JM, Tripathi N. Child sexual abuse in India: Current issues and research. Psychol Stud. 2013;58(3):318-25. https://doi.org/10.1007/s12646-013-0198-6

54. Singh MM, Parsekar SS, Nair SN. An epidemiological overview of child sexual abuse. J Fam Med Prim Care. 2014;3(4):430-35. https://doi.org/10.4103/2249-4863.148139 PMid:25657958

55. Carson DK, Foster JM, Chowdhury A. Sexual abuse of children and youth in India an anthropological perspective. Orient Anthropol. 2014;14(2):149-170.

56. Loveleen K, Srinivas V, Kumar P. Study on Child Abuse: INDIA 2007. Ministry of Women and Child Development, Government of India; 2007.

57. Dummett M. Breaking the Silence: Child Sexual Abuse in India. Human Rights Watch; 2013.

58. Choudhury R. Understanding Family Life in India. Family Life Education in India: Perspectives, Challenges, and Applications; 2006. p. 31-57.

59. Gorey KM, Leslie DR. The prevalence of child sexual abuse: Integrative review adjustment for potential response and measurement biases. Child Abuse Neglect. 1997;21(4):391-8. https://doi.org/10.1016/s0145-2134(96)00180-9

PMid:9134267 
60. Dube SR, Anda RF, Whitfield CL, Brown DW, Felitti VJ, Dong M, et al. Long-term consequences of childhood sexual abuse by gender of victim. Am J Prev Med. 2005;28(5):430-8. https://doi. org/10.1016/j.amepre.2005.01.015

PMid:15894146

61. Engstrom M, El-Bassel N, Gilbert L. Childhood sexual abuse characteristics, intimate partner violence exposure, and psychological distress among women in methadone treatment. J Subst Abuse Treat. 2012;43(3):366-76. https://doi. org/10.1016/j.jsat.2012.01.005

PMid:22444420

62. Engstrom M, Winham K, Gilbert L. Types and characteristics of childhood sexual abuse: How do they matter in HIV sexual risk behaviors among women in methadone treatment in New York city? Subst Use Misuse. 2016;51(3):277-94. https://doi.org/10.3 $109 / 10826084.2015 .1058823$

PMid:26886405

63. Rassenhofer M, Zimmer A, Spröber N, Fegert JM. Child sexual abuse in the Roman Catholic Church in Germany: Comparison of victim-impact data collected through church-sponsored and government-sponsored programs. Child Abuse Neglect. 2015;40:60-7. https://doi.org/10.1016/j.chiabu.2014.11.013 PMid:25528952

64. Assari S, Lankarani MM. Violence exposure and mental health of college students in the United States. Behav Sci. 2018;8(6):53. https://doi.org/10.3390/bs8060053

PMid:29882926

65. Wijaya MT. Contesting the dominant discourse of child sexual abuse: Sexual subjects, agency, and ethics. Sex Cult. 2018;22(3):740-57. https://doi.org/10.1007/s12119-018-9506-6

66. Mulya TW. Contesting the dominant discourse of child sexual abuse: sexual subjects, agency, and ethics. Sex Cult. 2018;22(3):740-57. https://doi.org/10.1007/s12119-018-9506-6

67. Chan SL, Khodabakhsh S. Counseling childhood sexual abuse survivors: A case study of challenges experienced by professional counselors and childhood sexually abused clients in Malaysia. J Asia Pac Couns. 2020;10(2):1-9. https://doi. org/10.18401/2020.10.2.9

68. Elklit A. Treatment of Danish survivors of child sexual abuse a cohort study. Behav Sci. 2015;5(4):589-601. https://doi. org/10.3390/bs5040589 PMid:26690484

69. Easton SD. Understanding adverse childhood experiences (ACE) and their relationship to adult stress among male survivors of childhood sexual abuse. J Prev Interv Community. 2012;40(4):291303. https://doi.org/10.1080/10852352.2012.707446 PMid:22970782

70. Felitti VJ, Anda RF, Nordenberg D, Williamson DF, Spitz AM, Edwards V, et al. Relationship of childhood abuse and household dysfunction to many of the leading causes of death in adults: The adverse childhood experiences (ACE) study. Am J Prev Med. 2019;56(6):774-86. https://doi.org/10.1016/j.amepre.2019.04.001 PMid:9635069

71. Ports KA, Ford DC, Merrick MT. Adverse childhood experiences and sexual victimization in adulthood. Child Abuse Neglect. 2016;51:313-22. https://doi.org/10.1016/j.chiabu.2015.08.017 PMid:26386753

72. Hamelin C, Salomon C, Cyr D, Gueguen A, Lert F. Childhood sexual abuse and adult sexual health among indigenous Kanak women and non-Kanak women of New Caledonia. Child Abuse Neglect. 2010;34(9):677-88. https://doi.org/10.1016/j.chiabu.2010.02.004 PMid:20663555

73. Isa MF, Ng CG, Rashid RA, Habil MH, Sidi H, Ayu SM, et al.
Sexual behaviour among male methamphetamine and heroin dependents in selected areas in Malaysia. Sains Malaysiana. 2013;42(12):1819-26.

74. Kaiser E, Sinanan AN. Survival and resilience of female street children experiencing sexual violence in Bangladesh: A qualitative study. J Child Sex Abuse. 2020;29(5):550-69. https://doi.org/10.1080/10538712.2019.1685615 PMid:31692413

75. Wang YW, Heppner PP. A qualitative study of childhood sexual abuse survivors in Taiwan: Toward a transactional and ecological model of coping. J Couns Psychol. 2011;58(3):393. https://doi. org/10.1037/a0023522.supp

PMid:21574695

76. Batool SS, Abtahi A. Psychosocial impact of childhood sexual abuse: Perspective of victims. J Arts Soc Sci. 2017;4(2):36-48.

77. Chouliara Z, Narang J. Recovery from child sexual abuse (CSA) in India: A relational framework for practice. Children Youth Serv Rev. 2017;79:527-38. https://doi.org/10.1016/j. childyouth.2017.06.072

78. Eisenbruch M. His body is human, but he has a tiracchāna heart: An ethnographic study of the epigenesis of child abuse in Cambodia. Child Abuse Neglect. 2019;88:129-43. https://doi. org/10.1016/j.chiabu.2018.10.018 PMid:30502578

79. Jinich S, Paul JP, Stall R, Acree M, Kegeles S, Hoff XC, et al Childhood sexual abuse and HIV risk-taking behavior among gay and bisexual men. AIDS Behav. 1998;2(1):41-51.

80. Sun YP, Dong ZJ, Yi MJ, Sun DF. Childhood sexual abuses among 1307 adult students and analysis on results of Symptom Checklist-90 test. Zhonghua Er Ke Za Zhi. 2006;44(1):21-5. PMid:16623999

81. Karayianni E, Fanti KA, Diakidoy IA, Hadjicharalambous MZ, Katsimicha E. Prevalence, contexts, and correlates of child sexual abuse in Cyprus. Child Abuse Neglect. 2017;66:41-52. https://doi.org/10.1016/j.chiabu.2017.02.016 PMid:28238355

82. Maier T, Mohler-Kuo M, Landolt MA, Schnyder U, Jud A. The tip of the iceberg. Incidence of disclosed cases of child sexual abuse in Switzerland: Results from a nationwide agency survey. Int J Public Health. 2013;58(6):875-83. https://doi.org/10.1007/ s00038-013-0498-6 PMid:23884375

83. Li N, Zabin LS, Ahmed S. The childhood sexual abuse among youth in three Asian cities: Taipei, Shanghai, and Hanoi. Asia Pac J Public Health. 2015;27(2):NP1566-77. https://doi. org/10.1177/1010539512471968 PMid:23343644

84. Sinanan AN. The impact of child, family, and child protective services factors on reports of child sexual abuse recurrence. J Child Sex Abuse. 2011;20(6):657-76. https://doi.org/10.1080/ 10538712.2011.622354

PMid:22126109

85. Han IY, Lee Y, Yoo SK, Hong JS. Prevalence of and risk factors for male sexual abuse: The case of south korea. J Loss Trauma. 2011;16(1):84-101. https://doi.org/10.1080/15325024.2010.519290

86. Ahearn LA. How Can i Protect My Child From Sexual Assault? Available from: https://www.parentsformeganslaw.org/ prevention-child-sexual-abuse/. [ Last accessed on 2021 May 22].

87. Nickerson AB, Livingston JA, Kamper-DeMarco K. Evaluation of second step child protection videos: A randomized controlled trial. Child Abuse Neglect. 2018;76:10-22. https://doi. org/10.1016/j.chiabu.2017.10.001

PMid:28992513 